\title{
IMPROVED SORTING METHODOLOGY OF DATA-PROCESSING INSTRUCTIONS
}

\author{
Andrii Borovyi ${ }^{1)}$, Volodymyr Kochan ${ }^{2)}$, Theodore Laopoulos ${ }^{1)}$, Anatoly Sachenko ${ }^{2)}$ \\ 1) Electronics Lab, Physics Dept., Aristotle University, 54124, Thessaloniki, Greece \\ e-mail: aborovyi@physics.auth.gr, laopoulos@physics.auth.gr \\ ${ }^{2)}$ Research Institute for Intelligent Computer Systems, Ternopil National Economic University \\ 3, Peremoha Sq., 46020 Ternopil, Ukraine \\ e-mail: oko@tneu.edu.ua, as@tneu.edu.ua
}

\begin{abstract}
An improved classification methodology for sorting data-processing instructions for ARM7TDMI CPU core is presented in this paper. Main discussion here is related to the process of creating appropriate training sets for neural network (NN) based estimation of power consumption. We have proposed instructions' sorting methodology according to the binary instruction representation and the resources being used for the overall system model. Thus separate instructions groups are obtained for NN-based estimation of power consumption. Experimental results of the proposed method confirm successful usage of this sorting methodology for providing higher accuracy estimation of power consumption.
\end{abstract}

Keywords: Power consumption, ARM7TDMI, Data-processing, instructions, neural networks, sorting training set, power estimation.

\section{INTRODUCTION}

One of the problems, which require detailed analysis during the design of embedded and autonomous systems, is power-aware design. Latest publications in this area [1], [2] describe the need for the accurate power consumption estimation not only during the design process, but as well as during the normal usage of the final devices. Important advantage of this research is possible power-aware design not only for the hardware, but for the software also. Proposed approach is based on that well-known fact, that power consumption of the CPU is determined by the currently running routines.

There are known power-optimization methods [3] [4] which are based on the fastest running of the routines and putting microprocessor into powersaving mode (usually, by disconnecting unused blocks of the CPU). These methods are highly effective when the program must run few times, and it doesn't involve detailed analysis of CPU's analysis of operation according to the powerconsumption.

In a previous publication of the same group [5] different measurement issues have been presented, along with results of measurement data analysis [6]. The estimation methodology was mainly presented in these papers, while the explicit data representation of the instructions was not examined. Consequently the error level of the analysis remained higher that it was expected, mainly due to the simple representation approach for the instructions' information. By contrast, an improved data sorting and analysis methodology will be represented in this paper aiming to complete the approach of using neural networks for the software-related power consumption estimation [7].

\section{ANALYSIS}

Artificial neural networks are proposed to be used in the present approach. The well known ARM7TDMI CPU core will be used as a test bench in this work. The power consumption for all types of instructions within its 32-bit set will be explored. Data-processing instructions are forming the biggest instruction group within the ARM7TDMI ARM Instruction Set, as well as the most-used during the regular routines usage [8]. That's why these instructions must be analysed in first-time order.

In order to minimize amount of real measurements, and avoid using only simulations, different data-estimation methods have been used during previous work of the Ukrainian group of 
authors [9]. The estimation of the accuracy of the calculations and testing is complex, because the information about the measurement methodology is not very clear [10]. According to the results of the previous work it was decided to use preliminary sorting methodology related to the maximum available data (maximum bits amount, that are used during the instruction forming, processing and execution) as well as grouping various instructions within separate subgroups, according to their power consumption performance. Because of these reasons the total amount of required real measurements is expected to decrease drastically.

The term "base power consumption" has been proposed in [10] for each instruction for simplifying estimations of the mathematical model development. It refers to power consumed by CPU during the execution of the instruction with its minimum values (zero values). Therefore, the appropriate amount of "basic" instructions has been written, according to the [7]. These instructions either described zero immediate values within the instruction or operated with the registers, which internal values has been equal to zero.

We have been trying to estimate power consumption of the CPU, using the approach that has been described above for the present research as well. Also we are taking into account only operation code and addressing mode, because all other values remained the same and didn't change. Doing so, all instructions of interest obtained decimal values (from 0 to 15 ) according to their OpCode value. Addressing modes were enumerated in the same way, but according to the order as mentioned in [10]. Power consumption estimation has been implemented within the MATLAB environment using "nntoolbox package", and neural networks correspondingly. Yet, the results became unstable (maximum estimation error rapidly changed) and unacceptable (error changed from $10 \%$ to $1000 \%)$. Thus, extra research effort on a more efficient representation of obtained data is required.

Analysis of the obtained data has shown that internal features of the data representation are not taken into account during data processing. These features should be considered during the analysis of the instructions' binary code that is processing by the CPU core. All the instructions within this research are existent and valid for the 32-bit mode of the CPU (ARM mode) and their length is 32bits. Therefore the detailed analysis of instructions' internal representation has been provided (with the disassembler that accompanies the ARM Developer Suite) and common bits were marked.

The analysis [9] discovered that gathering instructions into separate groups is an effective way in terms of power consumption. Using this fact, the following three groups were created (according to the second operand value):

1. instructions with the second operand as immediate value;

2. instructions with the second operand as register or register shifted to the immediate value;

3. instructions with the second operand as register or register shifted to the register.

We should note that proposed method for sorting instructions excludes a description of the redundant data, allowing instructions definition in fixed format, and decreasing noises amount for NN (Table 1).

Table 1. Preliminary results of CPU power consumption during the data-processing instructions

\begin{tabular}{|l|c|}
\hline Parameter & Value \\
\hline Architecture of neural network & $6-8-1$ \\
\hline Vectors amount in training set & 12 \\
\hline Vectors amount in verification set & 12 \\
\hline Mean square error & $10-8$ \\
\hline Training epochs & 20785 \\
\hline Average training error & $0,0 \%$ \\
\hline Maximum training error & $0,1 \%$ \\
\hline Average estimation error & $2,7 \%$ \\
\hline Maximum estimation error & $5,2 \%$ \\
\hline
\end{tabular}

Despite of the acceptable value of NN's average estimation error, as in shown in Table 1, the maximum estimation error remains high, therefore training set must be analysed further in order to remove data with noise. The ARM Assembler syntax as well as data binary representation has been taken into account during the creation of the new training set. According to the syntax the three following subgroups were created:

1. instructions where all three operands are used for data processing;

2. instructions where both the destination and the second operands only are used for data processing;

3. instructions where both the first and the second operands only are used for data processing.

Thus, after the analysis nine subgroups for dataprocessing instructions were formed, according to the syntax and the maximum length of the second operand to the each of those has its own unique structure and requires separate $\mathrm{NN}$ for power estimation.

Such grouping may be seen in Table 2 . 
Table 2. Data-processing instructions, according to predefined criteria

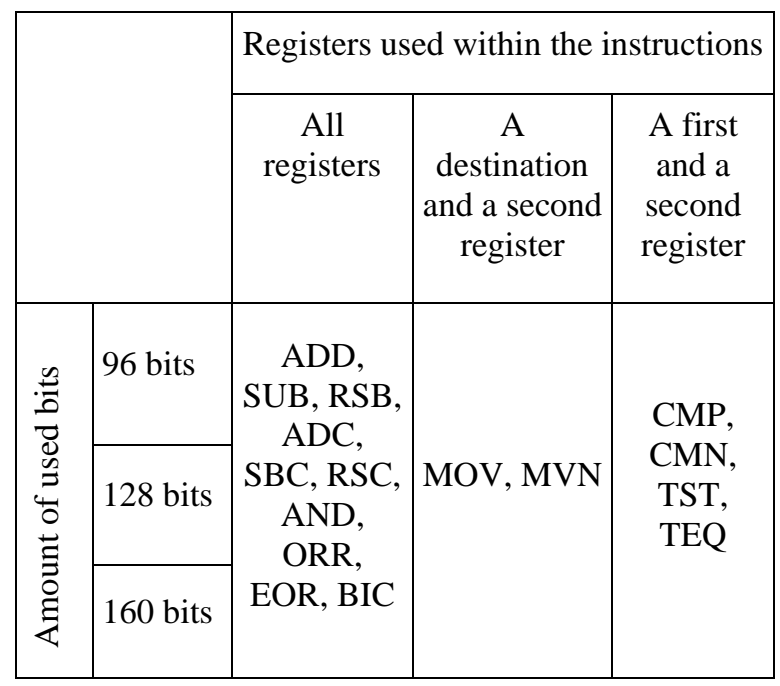

\section{ARCHITECTURE OF NEURAL NETWORK}

According to the above analysis, the appropriate architecture for the neural network is developed. The input layer has 6 neurons, the hidden one -4 neurons and output layer has 1 neuron. Its output value will correspond to the power consumption of the CPU. Multilayer perceptron has been used as non-linear activity function. This model is very simple, as well as universal model for the estimations [11], [12]. Output value for the threelayer perceptron can be defined with the next equation:

$$
y=F_{3}\left(\sum_{i=1}^{N} w_{i 3} h_{i}-T\right),
$$

where $N$ - amount of the neurons at hidden layer, $w_{i 3}$ - weight of the synapse from hidden neuron $i$, to the output layer, $h_{i}$ - output value of the neuron $i, T$ - threshold for output neuron, and $F_{3}$ activity function for the output neuron.

Output value for hidden neuron $j$ can be estimated with the next equation:

$$
h_{j}=F_{2}\left(\sum_{i=1}^{M} w_{i j} x_{i}-T_{j}\right) \text {, }
$$

where $w_{i j}$ - weight coefficient for connection between input neuron $i$ and hidden neuron $j, x_{i}-$ input values, and $T_{j}$ - threshold of hidden neuron $j$. Activity function for the hidden layer will be sigmoid, and activity function for the output layer will be linear function with the $k$ coefficient [13].

The back-propagation algorithm [14] has been used as training algorithm. This algorithm is based on gradient decreasing method and provides iterating procedure for renewal of weights and thresholds for each vector $p$ from the training set:

$$
\begin{gathered}
\Delta w_{i, j}=-\mathbf{\alpha} \frac{\partial E^{p}(t)}{\partial w_{i, j}(t)}, \\
\Delta T_{j}(t)=-\boldsymbol{\alpha} \frac{\partial E^{p}(t)}{\partial T_{j}(t)},
\end{gathered}
$$

where $\boldsymbol{\alpha}$ training coefficient, $\frac{\partial E^{p}(t)}{\partial w_{i, j}(t)}$ and $\frac{\partial E^{p}(t)}{\partial T_{j}(t)}$

- function error gradients at iteration $t$ for training vector $p, p \in\{1, \ldots, P\}$, where $P$ - size of the training set.

Mean-square error for iteration $t$ is estimating according to the next equation:

$$
E^{p}(t)=\frac{1}{2}\left(y^{p}(t)-d^{p}(t)\right)^{2}
$$

where $y^{p}(t)$ is outputting value for iteration $t$, and $d^{p}(t)$ - target outputting value for the training vector $p$.

During the training process, overall error is estimated as:

$$
E(t)=\sum_{p=1}^{P} E^{p}(t)
$$

The steepest descent method for calculating the learning rate [13] is used for removing the classical disadvantages of the back propagation error algorithm. Thus, the adaptive learning rates for the logistic and linear activation functions are given, respectively, by: 


$$
\begin{gathered}
\mathrm{\alpha}(t)=\frac{4}{\left(1+\left(x_{i}^{p}(t)\right)^{2}\right)} \\
\frac{\sum_{j=1}^{N}\left(\mathrm{\gamma}_{j}^{p}(t)\right)^{2} h_{j}^{p}(t)\left(1-h_{j}^{p}(t)\right)}{\left(\sum_{i=1}^{N}\left(\left(\mathrm{~V}_{j}^{p}(t)\right)^{2} h_{j}^{p}(t)\right)\right)^{2}\left(1-h_{j}^{p}(t)\right)^{2}} \\
\alpha(t)=\frac{1}{\sum_{i=1}^{N}\left(h_{i}^{p}(t)\right)^{2}+1},
\end{gathered}
$$

where, for the training vector $p$ and iteration $t$, $\mathrm{Y}_{j}^{p}(t)$ - is the error of neuron $j$ and $h_{j}^{p}-$ is the input signal of the linear neuron.

The error of neuron $i$ with logistic activation function can be determined by the expression:

$$
\mathrm{Y}_{j}^{p}=\sum_{j=1}^{N} \mathrm{Y}_{3}^{p}(t) w_{i 3}(t) h_{i}^{p}(t)\left(1-h_{j}^{p}(t)\right)
$$

where $\mathrm{Y}_{3}^{p}(t)=y^{p}(t)-d^{p}(t)$ is the error of the output neuron, $w_{i 3}-$ is the weight of the synapses between the neurons of the hidden layer and the output neuron.

The described algorithms of $\mathrm{NN}$ have been implemented in routine for estimating power consumption of the CPU.

\section{NN VERIFICATION RESULTS}

The next step in the described procedure is to form groups according to the proposed method of the sorting instructions and taking into account available data about CPU's "basic" power consumption during the data-processing instructions execution:

1. Arithmetic-logic instructions instructions):

1.1. 96 bits -10 vectors;

1.2. 128 bits -60 vectors;

1.3. 160 bits -40 vectors.

2. Movement instructions (2 instructions):

2.1. 64 bits -2 vectors;

2.2. 96 bits -12 vectors;

2.3. 128 bits -8 vectors.

3. Comparing and testing instructions (4 instructions):

3.1. 64 bits -4 vectors;
3.2. 96 bits -24 vectors;

3.3. 128 bits -16 vectors.

Taking into account, that maximum available data for creating training and verification sets have been obtained for subgroup 1.3, it has been decided to perform power analysis for this subgroup.

Research results are represented in Table 3.

Table 3. NN-prediction of power consumption

\begin{tabular}{|l|l|}
\hline Vectors amount in training set & 20 \\
\hline Vectors amount in verification set & 20 \\
\hline Mean square error & $10-6$ \\
\hline Training epochs & 11444 \\
\hline Average training error & $0,20 \%$ \\
\hline Maximum training error & $0,60 \%$ \\
\hline Average estimation error & $1,80 \%$ \\
\hline Maximum estimation error & $5,30 \%$ \\
\hline Architecture of neural network & $2-13-1$ \\
\hline
\end{tabular}

Comparing results, provided in Tables 1 and 3, it is observed, that proposed sorting methodology provides decreasing of average predicting error up to $1,8 \%$ that is much better than in the previous results.

Partial output of the NN verification is provided in Table 4.

Table 4. Partial verification results for the NN

\begin{tabular}{|c|c|c|c|c|}
\hline Number & Real & Predicted & Abs. Err. & Rel. Err. \\
\hline 22 & 0,926 & 0,894 & $-0,032$ & $3,50 \%$ \\
\hline 23 & 1,01 & 1,066 & 0,054 & $5,30 \%$ \\
\hline 24 & 0,82 & 0,810 & $-0,005$ & $0,60 \%$ \\
\hline 25 & 1,12 & 1,118 & 0,002 & $0,01 \%$ \\
\hline
\end{tabular}

\section{CONCLUSIONS AND FUTURE WORK}

Improved data processing methodology has been proposed. This methodology is based on accounting Assembler syntax of the instruction as well as used resources. Gathering instructions into groups provided exclude extra "noises" from the data, describing only required resources. NN-based estimation of power consumption confirmed decreasing of average estimation error. Increasing of maximum estimation error for $0,1 \%$ can be explained with the absence of exact description of all the fields of the instructions (in terms of data).

Future research will be focused on estimating power consumption while the instructions will be in different states, not only in "basic" one. It may implement deeper usage of $\mathrm{NN}$, providing more 
accurate estimation.

\section{AKNOWLEDGEMENTS}

This research has been performed under the financial support from the State Scholarship Foundation of Greek Republic (IKY, www.iky.gr) within the program of Scholarships for Postgraduate/Postdoctoral Studies in Greece, Subprogram: Collection of Research Data.

\section{REFERENCES}

[1] Nikolaidis S., Chatzigeorgiou A., and Laopoulos Th. Developing an Environment for Embedded Software Energy Estimation. Computers, Standards and Interfaces, Vol. 28, N. 2, 2005.

[2] Kavvadias N., Neofotistos P., Nikolaidis S., Kosmatopoulos C., and Laopoulos Th. Measurements Analysis of the SoftwareRelated Power Consumption of Microprocessors. IEEE Transactions on Instrumentation and Measurement, Vol. 53, N. 4, 2004.

[3] Carlo Brandolese, William Fornaciari, and Fabio Salice. Ultra Low-Power Electronics and Design, chapter Source-Level Models for Software Power Optimization, pages 156-171. Politecnico di Torino, Italy, 2004.

[4] M. F. Jacome, A. Ramachandran. Power Aware Embedded Computing. Embedded Systems Handbook Zurawski, R. (ed.) CRC Taylor \& Francis, 2006, pp. 16-1 - 16-17.

[5] A. Borovyi, V. Konstantakos, V. Kochan et al. Analysis of CPU's instructions energy consumption device circuits. Proceedings of the fourth IEEE international workshop on Intelligent Data Acquisition and Advancing Computing Systems (IDAACS 2007), Dortmund, Germany, September 9-11, 2007. pp. 42-47. - ISBN 978-1-4244-1347-8

[6] A. Borovyi, V. Konstantakos, V. Kochan et al. Using Neural Network for the Evaluation of Power Consumption of Instructions Execution. Proceedings of the Fifth International Instrumentation and Measurement Technology Conference (I2MTC'2008). Vancouver Island, Victoria, British Columbia, Canada, May 1215, 2008. pp. 676-681.

[7] ARM Limited, editor. ARM Architecture Reference Manual. Number ARM DDI 0100I. ARM Limited, 2007.

[8] S. Segars. ARM7TDMI Power Consumption. IEEE MICRO, 17(4):12-19, July- Aug. 1997.

[9] A. Borovyi, O. Havryshok, V. Kochan, Z. Dombrovsky. Development problems of the CPU power consumptionm model. Proceedings of the 10th International Scientific Conference
"Modern Information and Electronic Technologies”, May 18-22 2009, Ukraine. Vol. 1, p. 157. (in Ukrainian).

[10] Nikolaidis S., Kavvadias N., Laopoulos T., Bisdounis L., Blionas S. Instruction-level energy modeling for pipelined processors. Journal of Embedded Computing (special issue on Low-Power Design), Cambridge International Science Publishing (CISP), No. 3, 2004.

[11] K. Hornik, M. Stinchcombe, and H. White. Multilayer feedforward networks are universal approximators. Neural Networks. (No. 2) (1989). pp. 359-366.

[12] Simon Haykin. Neural Networks and Learning Machines. 3rd Edition, Prentice Hall, 2008. $936 \mathrm{p}$.

[13] V. Golovko. Neural Networks: training, models and applications. Radiotechnika. Moscow, 2001, P. 256. (In Russian).

[14] D. Rumelhart, G. Hinton, R. Williams. Learning representation by back-propagation errors. Nature. (323) (1986) pp. 533-536.

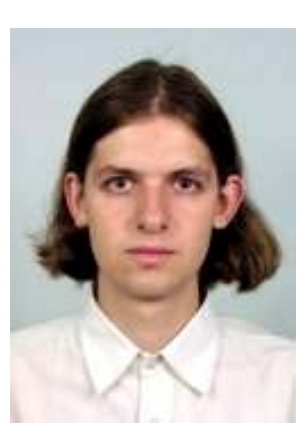

Andrii Borovyi has graduated Ternopil State Technical University as master in Information Control Systems and Technologies, 2006. Now he is a Ph.D. student and works in Department of InformationComputing System and Control as lecturer and in Research Institute for Intelligent Computer Systems, Ternopil National

Economic University.

His research interests inlude: embedded microprocessors, neural networks, systems for energy measurement of pulse consumers, programming languages $C$, Assembler.

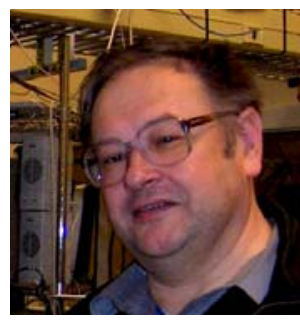

Volodymyr Kochan, Ph.D. of Measurement Engineering, Associate Professor, was born in 1951 in Lviv. In 1973 he received $B$. Eng. Electrical Engineering at Lviv Polytechnic Institute, Ukraine. In 1989 he obtained Ph.D. of Electric and Magnetic Instrumentation at Kiev Polytechnic Institute, Ukraine. Now he works as Associate Professor of Department of Specialized Computing Systems and Director of Research Institute for Intelligent Computer Systems of Ternopil National Economic University; Department of Computer Science of Ternopil State Technical University; Department of Electrical devices of 
Technical college of Ternopil State Technical University; Instructor of the practical course on microprocessor application.

His research area includes: Sensor Intelligent System; Distributed Sensor Network; Computer based Intelligent Measurement and Control Systems; Intelligent Controllers for Automated and Robotic Systems in Industry; Sensor Systems Calibration and Verification.

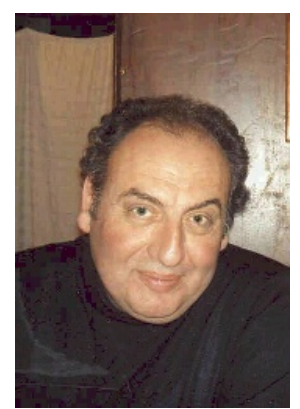

Theodore Laopoulos, is Associate Professor at the Electronics Lab., Physics Department, Aristotle University of Thessaloniki, Greece, where he is leading the "Systems for Electronic Measurements and Automation" group. His interests are in the fields of: Instrumentation Circuits and Systems, Measurement Systems and Techniques, Sensor Interfacing and Control Electronics, Applications of Microcontroller Systems, and Development of Education on Electronic Instrumentation.

Dr. Laopoulos has published over 100 papers in international scientific journals and conferences, and has supervised one PhD work and many Master theses in related subjects. He has served as leader (coordinator) in 11 Greek and European research projects and as senior researcher in certain others. Dr. Laopoulos is an IEEE senior member, Associate Editor of the IEEE Transactions on Instrumentation and Measurement, Academic coordinator of the Socrates/Erasmus program of the Physics Dept., and chairman of the Advisory Board of the "IDAACS" International Workshop on "Intelligent Data Acquisition and Advanced Computing Systems".

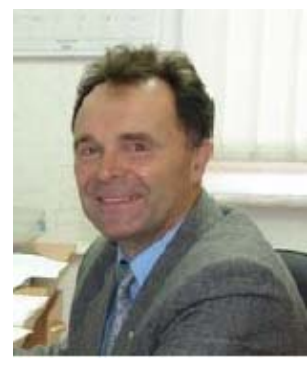
Anatoly Sachenko is Professor and Head of the Department of Information Computing Systems and Control and Research advisor of the Research Institute for Intelligent Computer Systems, Ternopil State Economic Uni- versity. He earned his B.Eng.

Degree in Electrical Engineering at L'viv Polytechnic Institute in1968 and his PhD Degree in Electrical Engineering at L'viv Physics and Mechanics Institute in 1978 and his Doctor of Technical Sciences Degree in Electrical and Computer Engineering at Leningrad Electrotechnic Institute in 1988. Since 1991 he has been Honored Inventor of Ukraine, since 1993 he has been IEEE Senior Member.

His main Areas of Research Interest are Implementation of Artificial Neural Network, Distributed System and Network, Parallel Computing, Intelligent Controllers for Automated and Robotics Systems. He has published over 430 papers in areas above. 